\title{
Cardiac arrest with pulseless electrical activity rhythm in newborn infants: a case series
}

\author{
Deandra Luong, ${ }^{1}$ Po-Yin Cheung, ${ }^{1}$ Keith J Barrington, ${ }^{2}$ Peter G Davis, ${ }^{3}$ Jennifer Unrau, ${ }^{4}$ \\ Shyamala Dakshinamurti, ${ }^{5}$ Georg M Schmölzer (10 ${ }^{6}$
}

'Department of Pediatrics,

University of Alberta, Edmonton, Alberta, Canada

${ }^{2}$ Department of Neonatology, Centre Hospitalier Universitaire Sainte Justine, Montreal,

Quebec, Canada

${ }^{3}$ Newborn Research, The Royal Women's Hospital, Melbourne, Victoria, Australia

${ }^{4}$ Department of Neonatology, University of Calgary Cumming

School of Medicine, Calgary, Alberta, Canada

${ }^{5}$ University of Manitoba

College of Medicine, Winnipeg.

Manitoba, Canada

${ }^{6}$ Department of Neonatology,

Royal Alexandra Hospital,

Edmonoton, Alberta, Canada

\section{Correspondence to}

Dr Georg M Schmölzer,

Neonatology, Royal Alexandra

Hospital, Edmonoton AB T5H

3V9, Canada;

georg.schmoelzer@me.com

Received 20 August 2018 Revised 21 January 2019 Accepted 3 February 2019

Published Online First

22 February 2019

Check for updates

(C) Author(s) (or their employer(s)) 2019. No commercial re-use. See rights and permissions. Published by BMJ.

To cite: Luong D, Cheung

P-Y, Barrington KJ, et al. Arch

Dis Child Fetal Neonatal Ed

2019:104:F572-F574.

\section{ABSTRACT}

The 2015 neonatal resuscitation guidelines added ECG to assess an infant's heart rate when determining the need for resuscitation at birth. However, a recent case report raised concerns about this technique in the delivery room. We report four cases of pulseless electrical activity during neonatal cardiopulmonary resuscitation in levels II-III neonatal intensive care units in Canada (Edmonton [ $n=3]$ and Winnipeg $[n=1]$ ). Healthcare providers should be aware that pulseless electrical activity can occur in newborn infants during cardiopulmonary resuscitation. We propose an adapted neonatal resuscitation algorithm to include pulseless electrical activity. Furthermore, in compromised newborns, heart rate should be assessed using a combination of methods/techniques to ensure accurate heart rate assessment. When ECG displays a heart rate but the infant is unresponsive, pulseless electrical activity should be suspected and chest compression should be started.

\section{INTRODUCTION}

Heart rate $(\mathrm{HR})$ is the most important clinical sign to evaluate compromised newborn infants and to guide resuscitation efforts in the delivery room. ${ }^{1}$ Increase in the newborn's HR remains the most reliable indicator of adequate ventilation. ${ }^{1}$ Until recently, HR in newborn infants at birth was assessed using: (1) palpation of the umbilical cord, (2) auscultation of the precordium and/or (3) pulse oximetry. ${ }^{1}$ In 2015 , the neonatal resuscitation guidelines changed their recommendation to suggest using ECG to assess an infant's HR immediately after birth. ${ }^{12}$ However, this weak recommendation was based on low-quality evidence, that is, observational data and small randomised trials. ${ }^{3-5}$

A recent case report of a newborn infant with hydrops fetalis raised concerns about the reliability of ECG. ${ }^{6}$ In addition, two case reports reported pulseless electrical activity (PEA) in newborn infants. One followed placement of a central catheter resulting in cardiac tamponade ${ }^{7}$ and the second occurred during neonatal resuscitation. ${ }^{8}$ Furthermore, we have reported two animal studies with cardiac arrest with PEA rhythm, where ECG displayed an HR ranging between $15 / \mathrm{min}$ and $80 /$ min in $23 / 54^{9}$ and $22 / 30^{10}$ asphyxiated piglets. The occurrence of cardiac electrical activity with no associated mechanical activity is called cardiac arrest with PEA rhythm. Depending on the electrical origin, PEA can be classified as sinus, atrial, junctional or ventricular. ${ }^{11}$ Furthermore, PEA is

\section{What is already known on this topic?}

- The 2015 neonatal resuscitation guidelines recommended ECG as a method to assess an infant's heart rate when determining the need for resuscitation at birth.

- A recent piglet study reported cardiac arrest with pulseless electrical activity rhythm in 23/54 asphyxiated piglets raising concerns about the reliability of ECG in newborn infants during cardiopulmonary resuscitation.

- A case of pulseless electrical activity in a newborn infant has been described after placement of a central catheter resulting in cardiac tamponade.

\section{What this study adds?}

- While ECG usually provides a rapid and accurate display of the heart's electrical activity, it does not prove the presence of effective cardiac function.

- Pulseless electrical activity can occur during neonatal resuscitation in the delivery room.

- During the management of the compromised infant, healthcare providers should not rely solely on ECG, and rather use a combination of tools to assess heart rate.

categorised as narrow QRS complex (70\% of cases) or wide QRS complex. ${ }^{11}$ Narrow QRS complex PEA might be due to cardiac tamponade, pulmonary embolism, tension pneumothorax or mechanical lung hyperinflation, ${ }^{11-13}$ while wide QRS complex PEA is more likely due to electrolyte disturbances, tachydysrhythmias, cardiomyopathy or cardiac ischaemia. ${ }^{11-13}$ In adults, PEA occurs in approximately $35 \%-40 \%$ of in-hospital arrests and $22 \%-30 \%$ of out-of-hospital cardiac arrests and is associated with a $2 \%-5 \%$ survival to discharge rate. ${ }^{12}$ Cardiac arrest with PEA rhythm has not previously been reported in newborn infants. However, since the 2015 guidelines changes, the authors of this case series have witnessed several newborn infants presenting with cardiac arrest with PEA rhythm.

\section{CASE PRESENTATIONS}

The authors have observed several cases at their respective institutions (The Royal Alexandra Hospital, Edmonton $[\mathrm{n}=3]$ and The Health 
Sciences Centre, Winnipeg $[n=1])$. The Royal Alexandra Hospital, Edmonton, is a tertiary perinatal centre admitting $\sim 350$ infants with a birth weight of $<1500 \mathrm{~g}$ annually and The Health Sciences Centre, Winnipeg, is a tertiary perinatal centre admitting $\sim 320$ infants with a birth weight of $<1500 \mathrm{~g}$ annually. In these cases, the clinical team observed cardiac arrest with PEA rhythm displayed on the monitor. Unfortunately, in several cases initiation of cardiopulmonary resuscitation was delayed because the clinical team relied on the displayed HR on the monitor instead of using other methods (eg, auscultation) to confirm the infant's HR.

\section{CASE 1}

A term infant was thought to suffer a skull and cervical spine injury following instrumental vaginal delivery. The infant was initially bradycardic but displayed PEA within the first 5 min of resuscitation. There was no detectable pulse, but an ECG HR of $60-75 / \mathrm{min}$ was observed. With chest compression and epinephrine administration, intermittent periods of return of spontaneous circulation (ROSC) occurred; however, they were followed by cardiac arrest with PEA rhythm. This sequence of ROSC and cardiac arrest with PEA rhythm occurred four times during the $50 \mathrm{~min}$ resuscitation. Placement of an umbilical arterial catheter displayed a pressure waveform during chest compressions but a flat line when chest compressions were stopped, despite presence of an electrical HR of 70/min. After $50 \mathrm{~min}$ of resuscitation, a stable pulse was established, and the infant was transferred to neonatal intensive care unit. Life support was discontinued after imaging confirmed extensive brain injury.

\section{CASE 2}

An extremely low birthweight infant was born via emergency caesarean section for antepartum haemorrhage. The infant's Apgar score remained at 1 at 1,5 and $10 \mathrm{~min}$ after birth. This represented the presence of an HR on ECG of 80-90/min. Initial mask ventilation, MR SOPA (M-mask adjustment, R-reposition airway, $\mathrm{S}$-suction mouth and nose, $\mathrm{O}$-open mouth, $\mathrm{P}-$ pressure increase, and A-airway alternative) and intubation were provided with no change in HR. After auscultation indicated cardiac arrest with PEA rhythm chest compressions, four doses of epinephrine and two boluses of $10 \mathrm{~mL} / \mathrm{kg}$ of normal saline were administered. However, this did not improve the infant's HR. During ongoing resuscitation, a chest X-ray revealed a large left-sided pneumothorax. Despite draining the pneumothorax, the $\mathrm{HR}$ remained unchanged. At $60 \mathrm{~min}$ after birth an arterial blood gas had a $\mathrm{pH}$ of 6.75 , an arterial $\mathrm{CO}_{2}$ of $119 \mathrm{~mm} \mathrm{Hg}$, a base excess of -18 and a lactate of $12.9 \mathrm{mmol} / \mathrm{L}$. At that time, a decision was made to discontinue resuscitation and the infant died.

\section{CASE 3}

A very low birthweight infant was born via caesarean section for fetal tachycardia. At the initial assessment, the infant's HR on the ECG was 70-80/min. However, auscultation detected an HR of $10-30 / \mathrm{min}$. This clinical picture indicated cardiac arrest with PEA rhythm. The infant's HR did not improve following mask ventilation, MR SOPA and intubation. The ECG continued to display a HR between $70 / \mathrm{min}$ and $80 / \mathrm{min}$, while auscultation measured a HR of 10-30/min. Despite chest compressions and four doses of epinephrine, the infant's HR did not improve and care was withdrawn at approximately $30 \mathrm{~min}$ after birth.

\section{CASE 4}

A premature infant with a birth weight of $1045 \mathrm{~g}$ was born vaginally with history of premature rupture of membrane for
8 weeks. At the initial assessment after birth, the infant's HR on the ECG was 40-50/min. However, auscultation detected no HR. This clinical picture indicated cardiac arrest with PEA rhythm. After mask ventilation, MR. SOPA and intubation the infant's HR remained 0/min, while the ECG continued to display 40-50/min. Despite chest compressions and two doses of epinephrine, the infant's HR did not improve, and care was withdrawn at approximately $20 \mathrm{~min}$ after birth.

\section{DISCUSSION}

This case series describes four newborn infants presenting with cardiac arrest associated with PEA on ECG. This is concerning as initiation of chest compressions might be delayed if clinicians rely solely on ECG display of HR. In these cases, hypoxia/ asphyxia, hypovolaemia due to antepartum haemorrhage and tension pneumothorax were the most likely causes for cardiac arrest with PEA. These examples are consistent with reports of cardiac arrest with PEA after hypoxia/asphyxia in newborn piglets $^{9} 10$ and in human neonates after placement of a central
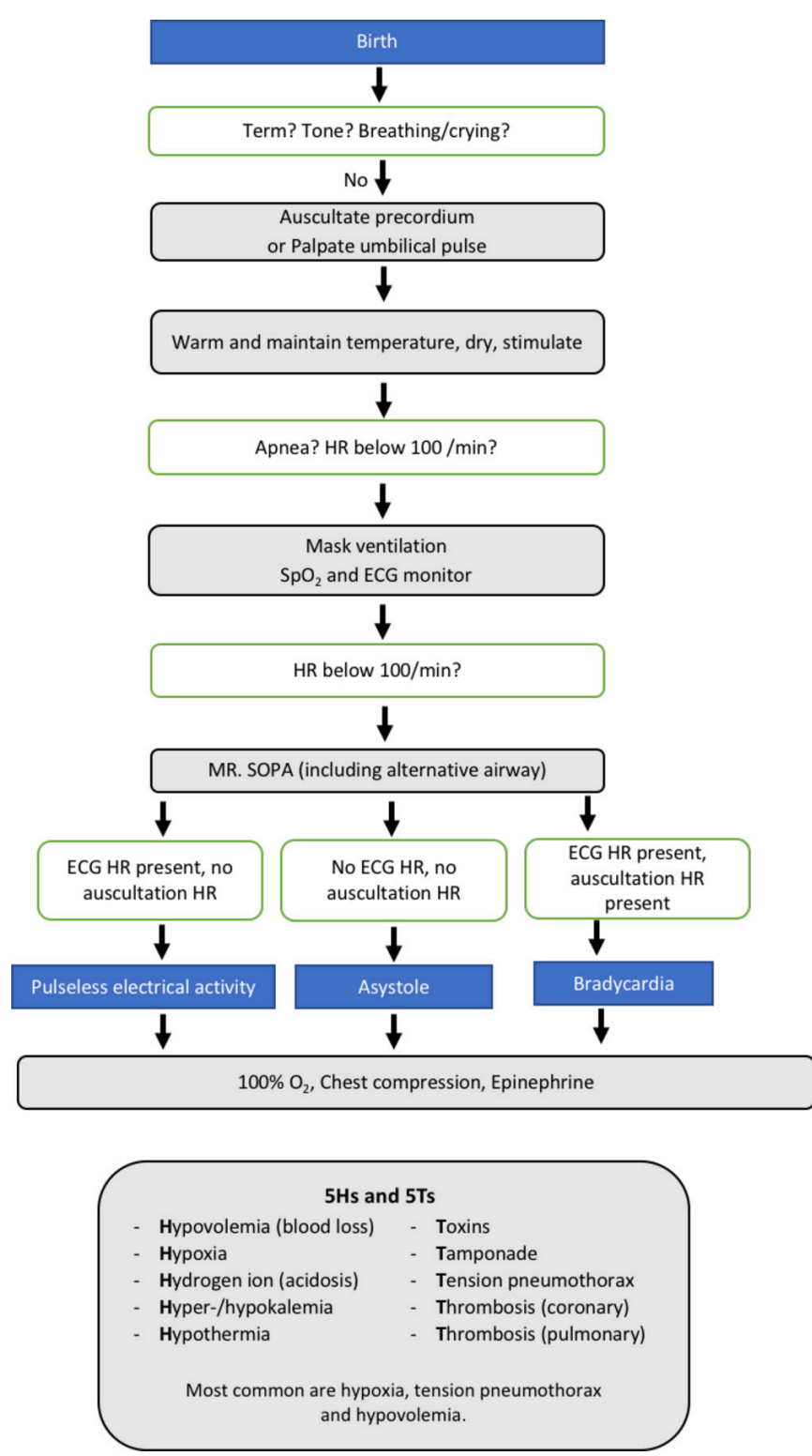

Figure 1 Proposed change in neonatal resuscitation algorithm. HR, heart rate. 
catheter resulting in cardiac tamponade ${ }^{7}$ and during neonatal resuscitation. ${ }^{8}$

The neonatal resuscitation programme (NRP) algorithm was developed for use in the delivery room to accommodate the specific physiological events that take place during transition. With the incorporation of ECG monitoring in the most recent edition, palpation of the umbilical cord and auscultation of the precordium may be overlooked. ${ }^{9}{ }^{13}$ Due to the possibility of cardiac arrest with PEA rhythm, relying solely on ECG is problematic, and other methods of clinical assessment should be implemented alongside ECG to ensure accurate assessment of cardiac function. Therefore, the NRP algorithm should be revised to distinguish between asystole, bradycardia and cardiac arrest with PEA. We therefore propose this adapted algorithm:

Step 1: assess infant's HR using auscultation or palpation Immediately after birth, the infant's HR should be assessed using auscultation or palpation (figure 1). The neonatal resuscitation guidelines state that 'Auscultation is performed by placing a stethoscope on the newborn's chest, and palpation is done by placing the umbilical cord between the thumb and the index finger'. 'The HR is assessed by listening or feeling the pulse for $6 \mathrm{~s}$ then multiplying this value by 10 to obtain a HR in beats per minute (beats $\min ^{-1}$ )'. ${ }^{12}$ We recognise that both methods have their pitfalls and are less accurate compared with pulse oximetry or ECG. ${ }^{1314}$ Clinical assessment underestimates the true HR with a mean difference (SD) between auscultation and palpation and ECG HR of $-14(21)$ and $-21(21) / \mathrm{min}^{-1}$, respectively. ${ }^{3}$ Both techniques are faster in obtaining an initial HR compared with pulse oximetry or ECG. ${ }^{13}{ }^{14}$ Newer techniques including Doppler ultrasound and digital stethoscopes might be useful; however, neither has been examined in asphyxiated infants. ${ }^{15} 16$

\section{Step 2: attach pulse oximeter and ECG to continuously monitor HR}

The neonatal resuscitation guidelines state that 'a pulse oximeter should be attached to the right hand/wrist ${ }^{17}$ and ECG-leads on clean areas of the skin on the chest'. ${ }^{2}$ Several studies have demonstrated that ECG measures HR faster and more reliably than pulse oximetry. ${ }^{45131418}$

\section{Step 3: assess HR prior to starting and during chest compressions}

Before starting, and periodically during ongoing resuscitation, evaluation of the HR should include a combination of methods (figure 1). If PEA is suspected, auscultation and/or palpation should be the cornerstone of HR assessment as pulse oximetry and ECG may be unreliable. In addition, point-of-care ultrasound to check for myocardial activity could aid in the diagnosis of PEA. ${ }^{16}$ If bradycardia is detected on clinical examination, ECG may be used to monitor HR.

\section{CONCLUSION}

Neonatal resuscitation guidelines have changed to suggest the use of ECG in the delivery room and in most cases ECG provides a fast and accurate measurement of a newborn's HR. However, clinicians need to be aware of PEA, during which clinicians may be falsely reassured that cardiac output is adequate. In general, auscultation and palpation are not as accurate as ECG or pulse oximetry, but they remain important clinical tools for HR assessment during cardiopulmonary resuscitation of newborn infants. We believe that each technique has a role and should be combined to allow adequate assessment of an infant's HR.

Acknowledgements We would like to thank the public for donating money to our funding agencies.

Contributors Conception and design: all authors. Collection and assembly of data: all authors. Analysis and interpretation of the data: all authors. Drafting of the article: all authors. Critical revision of the article for important intellectual content: GMS, P-YC, SD, JU, KJB, DL. Final approval of the article: all authors.

Funding GMS is a recipient of the Heart and Stroke Foundation/University of Alberta Professorship of Neonatal Resuscitation, a National New Investigator of the Heart and Stroke Foundation Canada and an Alberta New Investigator of the Heart and Stroke Foundation Alberta. PGD is supported by an NHMRC Practitioner Fellowship \#1059111. This research has been facilitated by the Women and Children's Health Research Institute through the generous support of the Stollery Children's Hospital Foundation.

Competing interests None declared.

Patient consent for publication Not required.

Ethics approval The ethics advisory committees at the University of Alberta in Edmonton and Manitoba in Winnipeg approved the publication of the information.

Provenance and peer review Not commissioned; internally peer reviewed.

ORCID iD

Georg M Schmölzer http://orcid.org/0000-0001-9798-2415

\section{REFERENCES}

1 Perlman JM, Wyllie J, Kattwinkel J, et al. Part 7: neonatal resuscitation. Circulation 2015;132:S204-41

2 Wyckoff MH, Aziz K, Escobedo MB, et al. Part 13: Neonatal Resuscitation: 2015 American Heart Association Guidelines update for cardiopulmonary resuscitation and emergency cardiovascular care. Circulation 2015;132:S543-60.

3 Kamlin CO, O'Donnell CP, Everest NJ, et al. Accuracy of clinical assessment of infant heart rate in the delivery room. Resuscitation 2006;71:319-21.

4 Katheria A, Arnell K, Brown M, et al. A pilot randomized controlled trial of EKG for neonatal resuscitation. PLoS One 2017;12:e0187730-8.

5 Katheria A, Rich W, Finer N. Electrocardiogram provides a continuous heart rate faster than oximetry during neonatal resuscitation. Pediatrics 2012;130:e1177-81.

6 Hodgson KA, Kamlin COF, Rogerson S, et al. ECG monitoring in the delivery room is not reliable for all patients. Arch Dis Child Fetal Neonatal Ed 2018;103:F88.1-F88.

7 Piastra M, Cavallaro F, Genovese O, et al. Double cooling for pericardial tamponade and cardiac arrest after rewarming in neonatal asphyxia. Resuscitation 2013;84:e83-4.

8 Sillers L, Handley SC, James JR, et al. Pulseless Electrical Activity Complicating Neonatal Resuscitation. Neonatology 2018;115:95-8.

9 Patel S, Cheung PY, Solevåg AL, et al. Pulseless electrical activity: a misdiagnosed entity during asphyxia in newborn infants? Arch Dis Child Fetal Neonatal Ed 2019;104:F215-F217.

10 Luong DH, Cheung PY, O'Reilly M, et al. Electrocardiography vs. Auscultation to Assess Heart Rate During Cardiac Arrest With Pulseless Electrical Activity in Newborn Infants. Front Pediatr 2018:6:S204-4.

11 Hauck M, Studnek J, Heffner AC, et al. Cardiac arrest with initial arrest rhythm of pulseless electrical activity: do rhythm characteristics correlate with outcome? Am J Emerg Med 2015;33:891-4.

12 Mehta C, Brady W. Pulseless electrical activity in cardiac arrest: electrocardiographic presentations and management considerations based on the electrocardiogram. Am J Emerg Med 2012;30:236-9.

13 Phillipos E, Solevåg AL, Pichler G, et al. Heart rate assessment immediately after birth. Neonatology 2016;109:130-8.

14 Dawson JA, Schmölzer GM, Wyllie J. Monitoring heart rate in the delivery room. Semin Fetal Neonatal Med 2018:23:327-32.

15 Kevat AC, Dawson J, Davis PG, et al. Evaluation of a digital stethoscope and smart device technology for assessment of heart rate in the newborn infant. Arch Dis Child Fetal Neonatal Ed 2015;100:F562-3.

16 Dyson A, Jeffrey M, Kluckow M. Measurement of neonatal heart rate using handheld Doppler ultrasound. Arch Dis Child Fetal Neonatal Ed 2017;102:F116-9.

17 Dawson JA, Kamlin CO, Vento M, et al. Defining the reference range for oxygen saturation for infants after birth. Pediatrics 2010;125:e1340-7.

18 Murphy MC, De Angelis L, McCollum D, et al. A randomised cross-over study of methods of acquiring ECG heart rate in newborns. Arch Dis Child Fetal Neonatal Ed 2017;102:F369-70. 